Check for updates

Cite this: RSC Adv., 2018, 8, 2660

\title{
Plasmono-magnetic material for precise photothermal heating $\dagger$
}

\author{
Mikhail Ladanov, + Surya Cheemalapati, $\$$ Hao Wang, Yuan Yuan, Piyush Koria \\ and Anna Pyayt (D)*
}

Noble metal nanoparticles have been extensively studied as photo-sensitive agents for photothermal cancer therapy. Precise control over the size and shape of the nanoparticles allowed strong optical absorption and efficient heat generation necessary for destroying a tumor to be achieved. However, one of the fundamental challenges of application of the nanoparticles towards photothermal cancer therapy is low specificity in the targeting tumor tissue in comparison with the healthy tissue and the resulting unfavorable biodistribution of the nanoparticles. Additional levels of control over particle distribution can be achieved by making the particles magnetic and using external magnets to control their accumulation in a tumor. Since the direct synthesis of particles with a magnetic core and a metallic shell limits the options for design and fine-tuning of plasmonic properties, the alternative approaches to the design of such materials have to be investigated. Here we propose and demonstrate a new design of a hybrid plasmono-magnetic material for photothermal heating created by grafting Au nanocages onto a surface of magnetic micro-beads. Next, we confirm its dual functionality in in vitro studies and show that individual hybrid particles can be magnetically controlled with a precision of a few micrometers and

Received 27th July 2017

Accepted 27th December 2017

DOI: $10.1039 / \mathrm{c} 7 \mathrm{ra0} 8276 \mathrm{~b}$

rsc.li/rsc-advances precisely destroy individual cells using plasmonic heating.
There is an increasing interest in the development of new cancer therapies in addition to existing chemo- and radiation therapies because of severe side-effects of the traditional methods. ${ }^{1}$ Light-based cancer therapy is a popular direction since it is minimally invasive and can be realized via different mechanisms ${ }^{2-6}$ including laser light ablation, ${ }^{3}$ photo dynamic therapy (PDT), ${ }^{4}$ and photo thermal therapy (PTT). ${ }^{5}$ Laser light ablation is conducted using an optical fiber delivering light directly to a tumor and thus heating it up to a high temperature. The main limitation of this therapy is the lack of selectivity and thus a high risk of damage to healthy tissues or organs. Photodynamic therapy helps to overcome this limitation by using photosensitizing agents that can be selectively introduced into a tumor to produce free radicals that destroy the diseased tissue. ${ }^{4,7}$ However, this therapy requires the presence of oxygen to be effective, ${ }^{4}$ and makes patients sensitive to light for a long time. ${ }^{6}$ The photosensitizing agents can be replaced with organic dyes absorbing light and generating heat. ${ }^{5}$ However, organic dyes normally have low photostability and are vulnerable to photobleaching. A major breakthrough in photo-thermal cancer therapy was the development of noble metal nanoparticles for

Department of Chemical and Biomedical Engineering, University of South Florida, Tampa, FL 33647, USA.E-mail: anna.pyayt@gmail.com

$\dagger$ Electronic supplementary information (ESI) available. See DOI: $10.1039 / \mathrm{c} 7 \mathrm{ra0} 276 \mathrm{~b}$

\$ Indicates equal contributions. plasmonic photothermal cancer therapy (PPTT). ${ }^{6}$ These nanoparticles are highly stable and their absorption cross-section is orders of magnitude higher than that of conventional dyes. ${ }^{8}$

Currently a lot of research is focused on Au nanocages that have localized surface plasma resonance (LSPR) peak optimized for extremely efficient near infrared (NIR) absorption at wavelengths least absorptive by human tissue., ${ }^{\mathbf{1} 9}$ Nanocages are

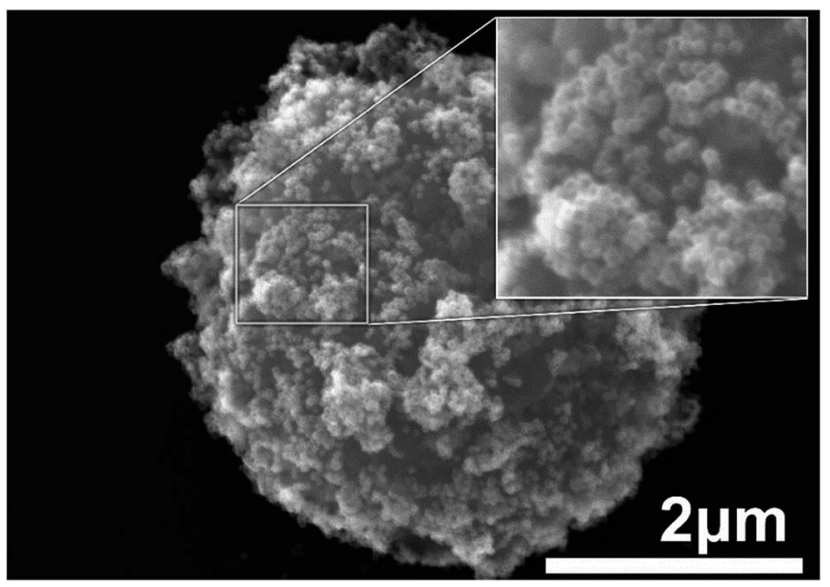

Fig. 1 SEM image of a single magnetic microbead decorated with nanocages. Inset shows a part of the bead imaged at higher magnification. Individual nanocages can clearly be seen on the surface of the microbead. 


\section{a)}

Microscope

objective

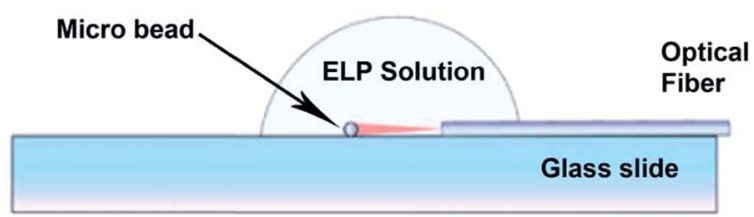

Light source
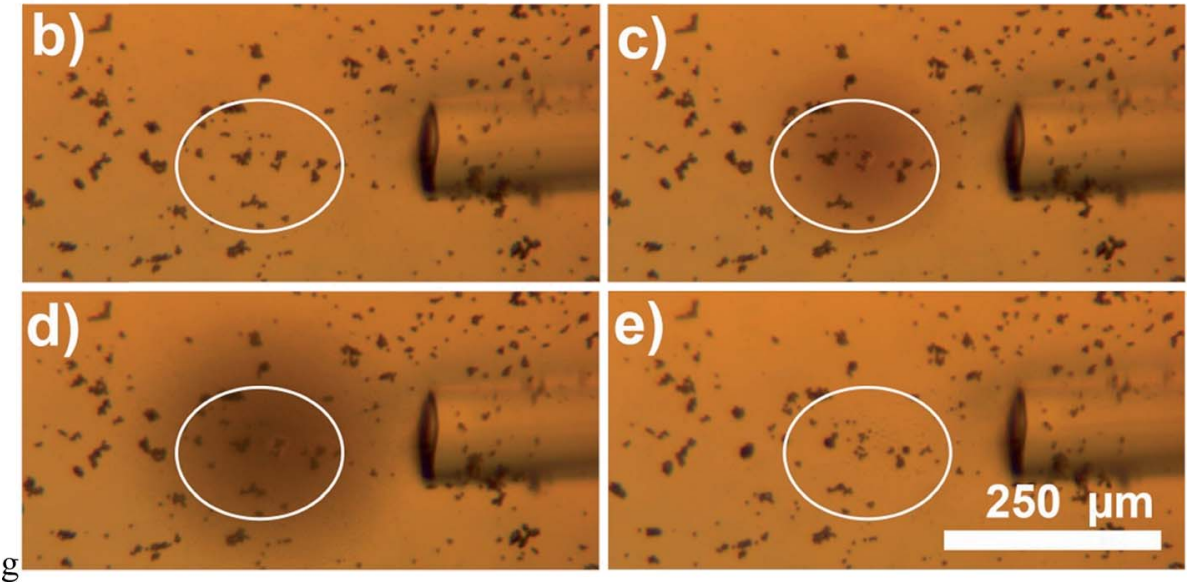

Fig. 2 Demonstration of the photothermal heat generation when the new composite material is exposed to NIR light. (a) The schematics of the proposed experiment. Microbead is shown as a small circle exposed to the light shining from the optical fiber. The whole assembly is immersed in a solution of elastin-like polypeptides and monitored under the microscope. (b) Optical microscopy image of a solution of ELPs with magnetic beads decorated with nanocages (dark particles). Optical fiber is immersed in the solution and is visible on the right side of the image. The light is turned off. (c) $\approx 30 \mathrm{~mW}$ laser light with wavelength of $808 \mathrm{~nm}$ is coupled into the optical fiber. The image is taken one second after the laser is turned on. (d) The steady state of the thermal profile is reached after $\approx 5$ seconds of the laser operation. (e) ELP with magnetic beads decorated with nanocages 5 seconds after the light is turned off. Based on the image luminosity analysis (see ESI† for details), the average temperature inside of the white circle goes up to $33.5^{\circ} \mathrm{C}$
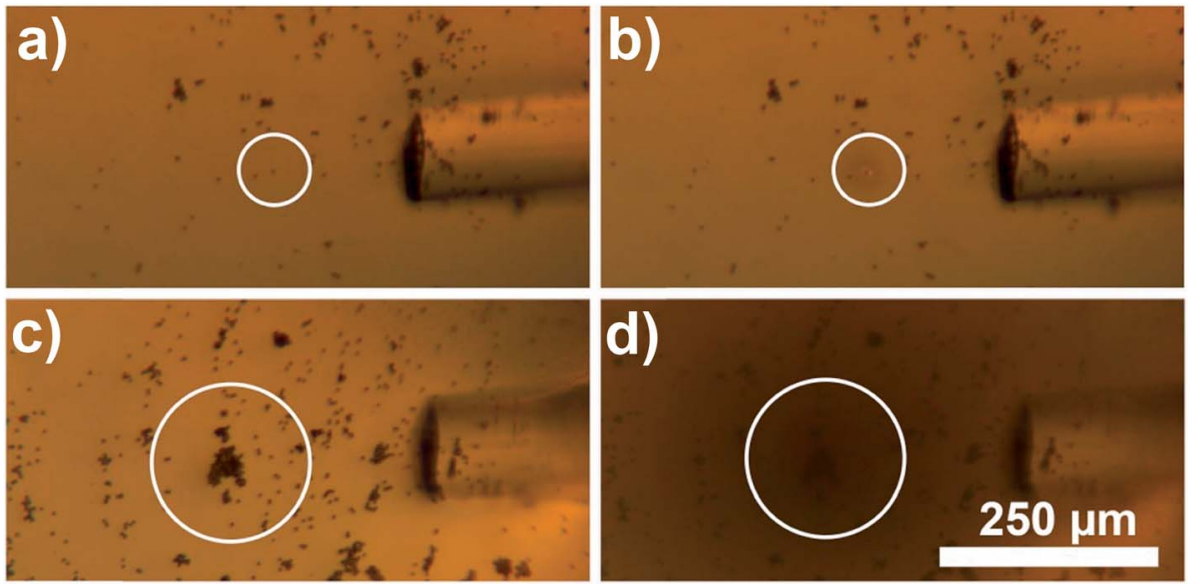

Fig. 3 Optical microscopy images showing heat generation vs. number of micro-beads exposed to NIR light. Parts (a) and (b) have low concentration of the Au nanocages decorated beads, (c) and (d) - higher concentration. (a) and (c) show the system when the laser is off, (b) and (d) after the light was turned on. In (b) an individual micro-bead is exposed to NIR light and small area around it is heated by $\sim 6{ }^{\circ} \mathrm{C}$ to $31^{\circ} \mathrm{C}$ (see the image luminosity analysis in ESI† for details). In (d) large clump of the micro-beads is exposed to NIR light and the temperature is increased above $36{ }^{\circ} \mathrm{C}$ in the whole field of view (above $40{ }^{\circ} \mathrm{C}$ near the beads). 
hollow, porous gold nanoparticles synthesized using galvanic replacement reaction between solutions containing gold precursor salts and silver nanostructures prepared through polyol reduction..$^{10}$ The effectiveness of Au nanocages for PPTT has already been successfully demonstrated in animal models. ${ }^{11}$ However, targeted delivery of Au nanocages to the tumors still stays as a fundamental limitation, since nanoparticles usually accumulate in all major organs. Therefore, additional controlling mechanisms that allow accumulation of photosensitive material in desired location are needed. This can be realized using magnetic particles and external magnets allowing concentrating the particles in a tumor. While hybrid nanoparticle can be synthesized simultaneously plasmonic and magnetic, this requires complex synthesis and limits control over optical properties of the particle. ${ }^{12-15}$

Here, we propose an alternative approach that allows making magnetic material while preserving unique plasmonic properties of the nanocages. It is based on hybrid particles that combine magnetic microbeads and $\mathrm{Au}$ nanocages. The microbeads are biocompatible, can be magnetically controlled from outside of the body, ${ }^{16,17}$ and have previously been used for targeted drug delivery, ${ }^{17}$ imaging, ${ }^{18}$ and other biomedical applications. ${ }^{19}$ In this work we proposed and demonstrated the composite particles consisting of magnetic microbeads covered with a layer of nanocages. After that we experimentally confirmed the magnetic properties of the new particle, as well as its efficient plasmonic heating via single-cell photothermal therapy.

The composite material was prepared according to the protocol described in Materials section. Briefly, suspensions of $\mathrm{Au}$ nanocages and AMS-40-10H SPHERO ${ }^{\mathrm{TM}}$ amino-coated magnetic particles were mixed together, and the composite material was formed by the electrostatic interactions between the nanocages and amine groups on the microbeads. ${ }^{20}$ Fig. 1 shows the SEM image of an individual magnetic microbead decorated by high density Au nanocages. The microbeads beads have a bumpy surface that results in increased surface area and larger number of adsorbed Au nanocages. Based on the SEM images, it is estimated that there are approximately 3000 nanocages present on the surface of a single microbead.

After attachment of the Au nanocages to the surface of the magnetic bead, the composite material still retain their plasmonic properties, and serve as an efficient heater upon exposure to the NIR light. In order to characterize its photothermal properties we used a new technique for visualization of temperature on micro-scale that we proposed earlier. ${ }^{21}$ Briefly, the composite material was mixed with the solution of elastinlike polypeptides (ELPs), that undergoes a phase transformation and change its absorption to visible light depending on its temperature. ${ }^{22}$ Derived from natural elastin, ELPs are composed of repeating blocks of penta-peptide with the sequence of Val-Pro-Gly-X-Gly, where $\mathrm{X}$ is a guest residue that can be any amino acid except proline. After mixing with ELP solution, magnetic micro-beads are exposed to NIR irradiation and microscopic heating is visualized via local change of optical properties observed under microscope. While experimental visualization of plasmonic heating on nano- and micro-scale is very challenging with traditional approaches, ${ }^{23-34}$ this method allows high resolution thermal field imaging in real time.

The schematic illustration of the experiment is shown in Fig. 2a. Solution of ELP was mixed with the Au nanocagesdecorated magnetic microbeads, and the NIR laser light was delivered to the solution through an optical fiber. Images were taken using a CCD camera attached to an optical microscope and equipped with infrared filter blocking light from the optical fiber. This way the changes in transparence and color of ELP solution become visible, and the excitation light is not seen in
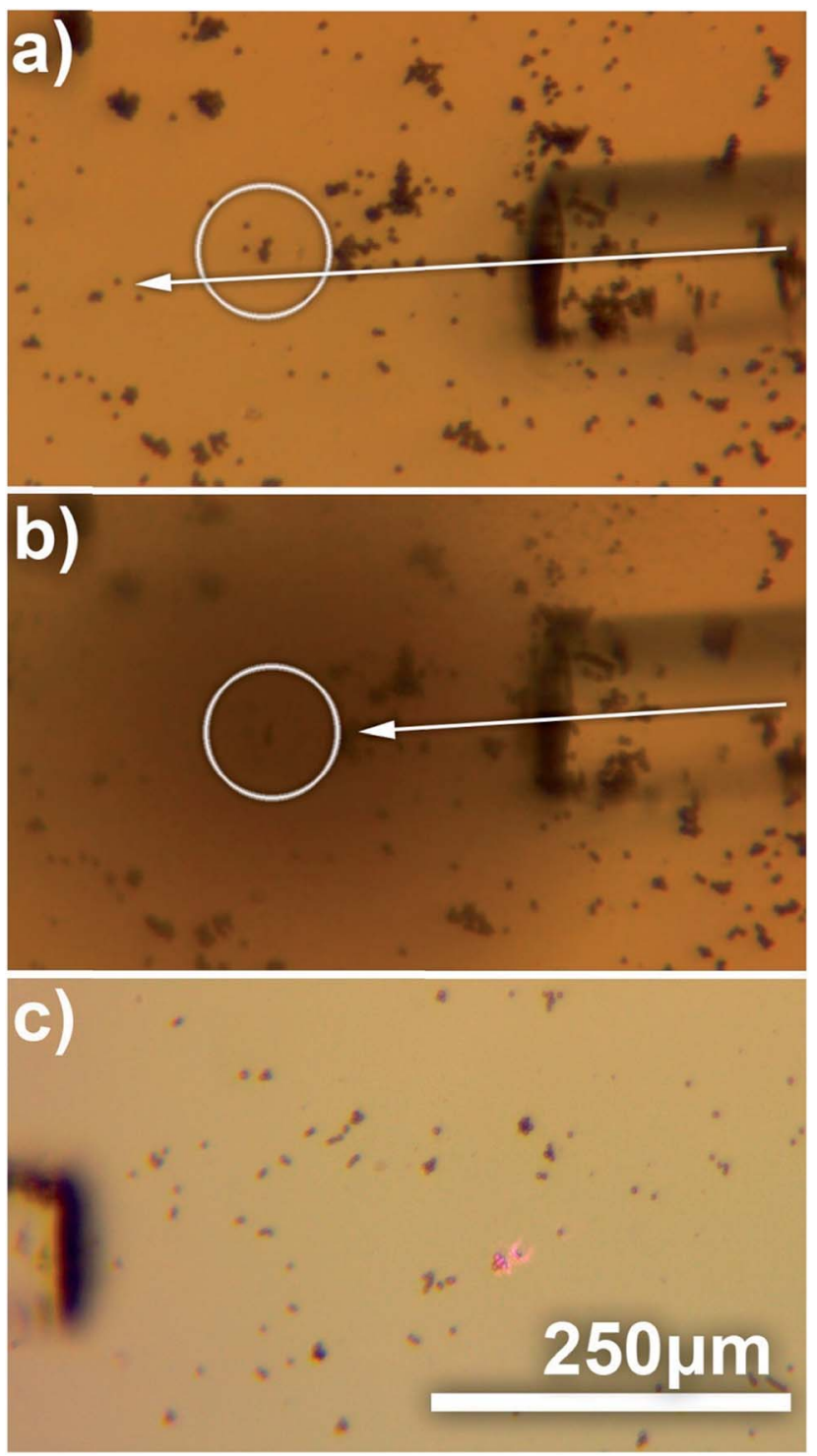

Fig. 4 Optical microscopy image demonstrating importance of alignment between the plasmono-magnetic material and the laser beam. In (a) small clump of Au nanocages-covered microbeads ( 10 beads) is slightly misaligned with the laser beam, and even though the laser is on, no heat is generated. In (b) optical fiber is aligned to the clump and the area inside of the white circle is heated on average above $34{ }^{\circ} \mathrm{C}$ (see the image luminosity analysis in ESI $\dagger$ for details). (c) Shows control experiment when the microbeads are not decorated with Au nanocages. IR filter is removed to demonstrate direct exposure of the micro-beads to the laser light. No heating is observed. 
the images. The darkness of the solution is directly correlated to a specific temperature.$^{18}$ Briefly, the luminosity of ELP solution observed under the microscope is related to the temperature of the solution via Boltzmann Sigmoid function, eqn (1).

$$
L=L_{2}+\frac{\left(L_{1}-L_{2}\right)}{1+\mathrm{e}^{\frac{\left(T-T_{t}\right)}{\mathrm{d} T}}}
$$

the constants $T_{t}$ and $\mathrm{d} T$ are obtained from the previously published calibration experiment. $L_{1}$ and $L_{2}$ are the maximum and minimum luminosities. The temperature change during plasmonic heating can be directly estimated from the luminosity of ELP solution using eqn (2).

$$
T=\ln \left[\frac{\left(L_{1}-L_{2}\right)}{\left(L-L_{2}\right)}-1\right] \mathrm{d} T+T_{t}
$$

In Fig. 2, the white circle outlines the areas exposed to the NIR laser light. The photothermal heating of $\mathrm{Au}$ nanocagesdecorated magnetic beads was monitored under microscope. Fig. 2b-e, demonstrates the heating properties of the composite material exposed to NIR laser irradiation in a time series. It can be noticed that the heating of just several beads in the center of the circle results in an increase of temperature in an area of approximately $200 \mu \mathrm{m}$ in diameter. The highest temperature in the central area was determined to be $\sim 33.5^{\circ} \mathrm{C}$ (more than $8{ }^{\circ} \mathrm{C}$ higher than surrounding). Furthermore, in Fig. 2, the size and darkness of the heated area is determined by equilibrium between the photothermal heat generation of the beads and heat dissipation into the surrounding medium. The large dark area and the high central temperature demonstrate that individual Au nanocages-covered microbeads still have efficient heating properties upon exposure to NIR light. Steady state heating profile was reached within 5-15 seconds after exposure to NIR light. When laser was off, the heat quickly dissipated into the surrounding medium. The system returned to its initial state with transparent ELP solution in approximately 5 seconds (Fig. 2d).

There are several approaches to control the heat generation and related temperature rise while using this novel magnetoplasmonic material. Control over laser intensity is the most straight forward way to manipulate the local temperature. By increasing the light intensity, Au nanocages-covered magnetic microbeads and the surrounding medium can be heated to higher temperature. However, for practical applications, such as photothermal cancer therapy, maximum laser power is limited by regulation due to the risk of damaging healthy tissue by a high intensity laser radiation.

The second approach to improvement of the photothermal heat generation is increase of the amount of light sensitive material exposed to the NIR light. As shown in Fig. 3, the amount of generated heat is directly related to the number of the hybrid particles exposed to NIR light. Fig. 3a and c were taken when the laser was off, and the background color was very uniform, indicating unvarying temperature distribution across the field of view. For both Fig. 3b and d, the laser light was kept at constant power $\approx 30 \mathrm{~mW}$. When a single bead was exposed to the light (Fig. 3b), the local temperature increase was approximately $6{ }^{\circ} \mathrm{C}$, while for a big clump of beads (Fig. 3d) the
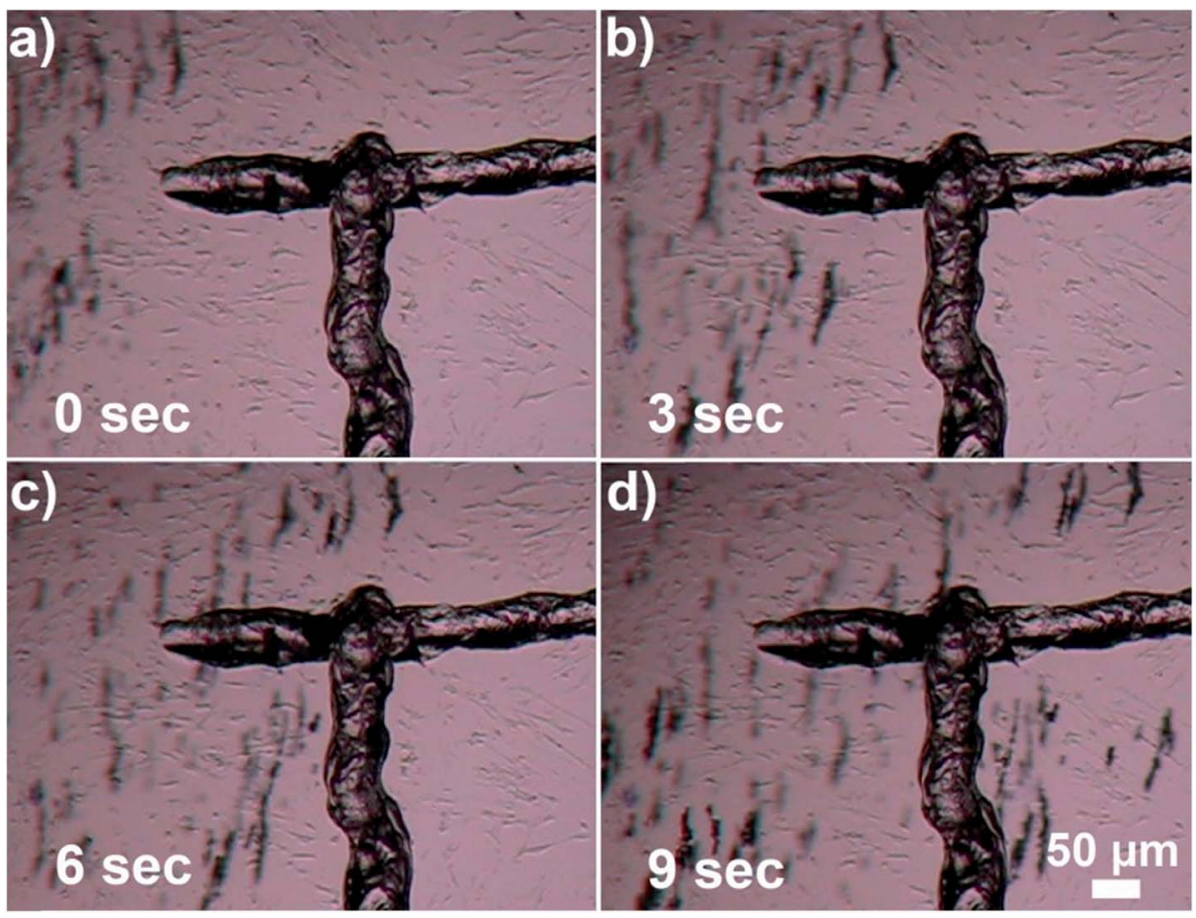

Fig. 5 Magnet-controlled movement of Au nanocages-covered magnetic microbeads towards T-marked area. (a) $1 \mu$ l solution of Au nanocages-decorated microbeads is dropped away from the marker. Next, the external magnet is placed approximately $1 \mathrm{~cm}$ away from the sample. (b)-(d) Gradual movement of the magnetic beads into the desired area. 
temperature in the whole field of view rose from $25{ }^{\circ} \mathrm{C}$ to more than $36{ }^{\circ} \mathrm{C}$, indicating a temperature increase of $11{ }^{\circ} \mathrm{C}$. This suggests that high concentration of $\mathrm{Au}$ nanocages-covered magnetic beads could be used to significantly increase temperature in tumor relative to the surrounding tissue during photothermal cancer therapy.

Additionally, use of this material allows achieving high selectivity and precision of heating. As shown in Fig. 4, the alignment of laser beam to the target composite particles greatly affects the heating efficiency. When a small clump of beads was just tens of micrometers misaligned from the center of the laser beam no heating was observed (Fig. 4a). When optical fiber was slightly moved to align the laser beam to the clump, the heat generation became very pronounced (Fig. 4b). This indicated that during photothermal therapy precise alignment of an external light to a tumor is very important. Furthermore, precise delivery of light using optical fibers allows controlling spatial precision of heating with the accuracy of few tens of micrometers.

Finally, in order to demonstrate that the heat generation by this new composite material is a result of localized surface plasmon resonance excitation of Au nanocages, and is not due to the absorption of NIR by magnetic micro-beads, a control experiment was conducted. Fig. 4c demonstrates, that without Au nanocages the microbeads do not generate heat, even when exposed to NIR light for 5 minutes. During that time there was no change in transparency of ELP solution which indicates no substantial heating, because of low absorption of NIR by the microbeads in absence of Au nanocages. When images of the control experiment were taken using a camera attached to the microscope, the IR filter was removed to make sure the laser beam was precisely aligned. Digital camera detected the scattered NIR light shining directly on the microbeads and displayed it via artificial pink color.

After the demonstration of heating properties of the new composite material, its magnetic properties were also confirmed by transporting the beads to a specific location using an external magnet. The magnet used in the experiments was neodymium disc magnets (NdFeB), $10 \mathrm{~mm}$ in diameter, thickness $2 \mathrm{~mm}$. The magnet was placed approximately $1 \mathrm{~cm}$ away from the sample. Fig. 5 shows migration of the Au nanocagesdecorated beads towards T-marked area in the Petri dish in
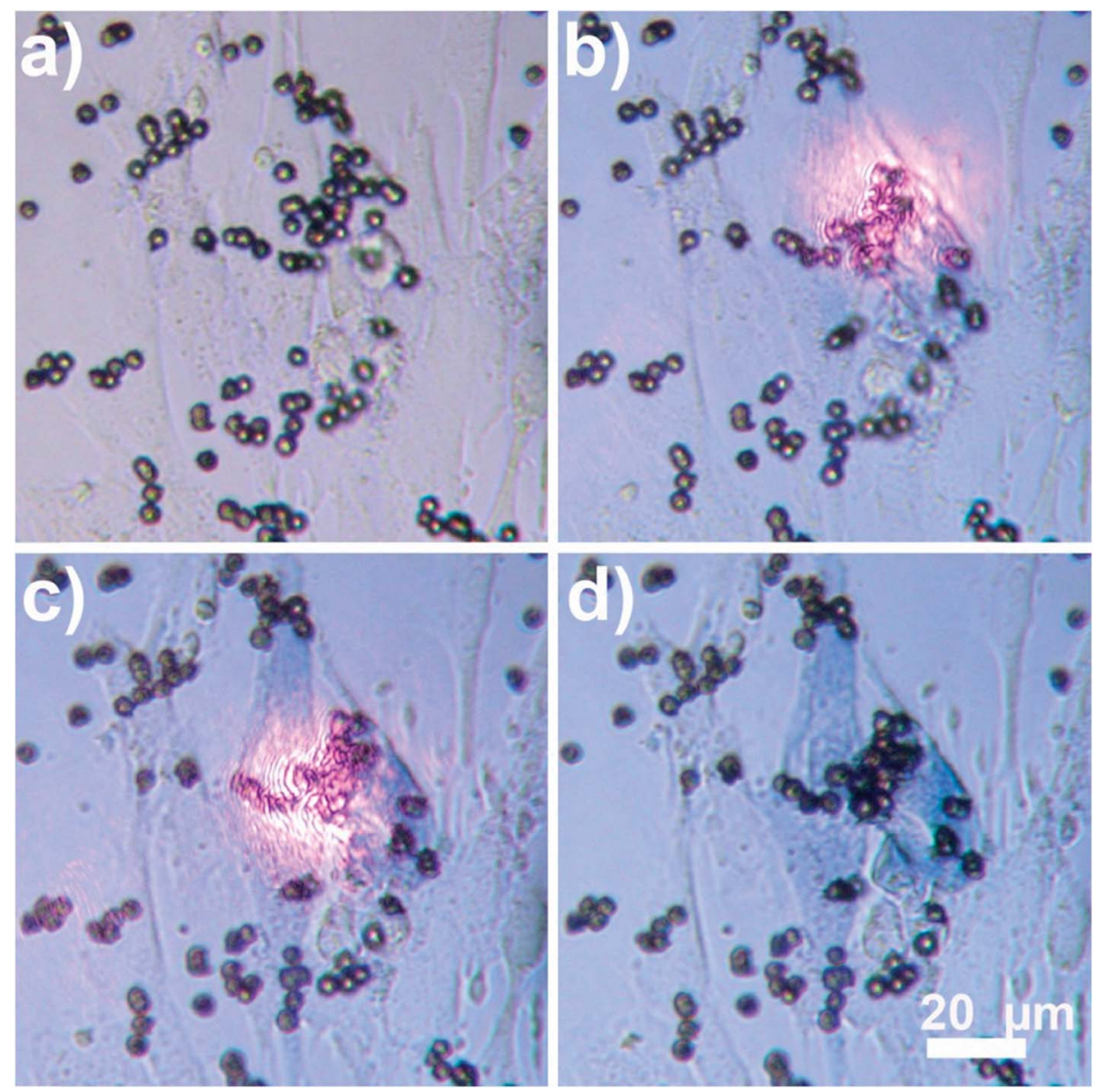

Fig. 6 Precise destruction of individual cells shown using Nikon microscope, $40 \times$ magnification. NIR light shines at a cluster of the nanocagecovered magnetic beads resting on top of a cell ( 15 microbeads). (a) Time -0 minutes (before light was on), (b) after 10 min of continuous light irradiation, one cell is dead and it is indicated by trypan blue dye staining dead cells blue. (c) After 20 min total, more dye diffuses inside the first cell. At the same time the laser beam is moved slightly to illuminate particles resting on top of the second cell. (d) 5 min later, laser is turned off, two dead cells are stained by trypan blue, all other cells are intact. 
response to the magnetic field. Initially $1 \mu \mathrm{l}$ of $1 \%(\mathrm{v} / \mathrm{v}) \mathrm{Au}$ nanocages-decorated magnetic beads suspension was dropcasted away from the marker. When an external magnet was placed on the right, the particles moved from the left to the right, into the T-marked area (Fig. 5a-d). This shows that in in vitro experiments $\mathrm{Au}$ nanocages-decorated microbeads can be magnetically controlled and delivered to any specific area covered with cells. As a result, the cells can be locally and controllably destroyed at desired location. As shown in Fig. 6a, there were multiple alive fibroblast cells. To distinguish between alive and dead cells, $100 \mu \mathrm{l}$ of trypan blue solution was added to the wells containing cells and allowed to diffuse in the cell medium for 3 minutes. Live cells appear transparent, and dead cells turn blue because of diffusion in of trypan blue. After that, the composite particles were steered towards the live cells, irradiated with NIR light and heated the cells. In Fig. $6 \mathrm{~b}$ and $\mathrm{c}$ two cells were sequentially heated by shining NIR light at composite particles resting on top of the cells. The targeted cells turned blue after the photothermal treatment due to diffusion of trypan blue, while the neighboring cells were unaffected. This demonstrates that $\mathrm{Au}$ nanocages-decorated microbeads are effective in heating and photothermal destroying the cells at a single cell level. For the constant power of $30 \mathrm{~mW}$, and $10 \mathrm{~min}$ time exposure the viability of cells and the precision of destruction is directly related to the number of beads exposed to the laser radiation located in close proximity to the cell. Specifically, single microbead covered with nanocages does not destroy the cell even after 30 min long exposure. Approximately 10 beads allow controllable destroying of a single cell without damaging the close cells. Finally, larger clamps of 50-100 of beads result in greatly increased temperature in area more than $500 \mu \mathrm{m}$ in diameter and corresponding simultaneous damage to many cells.

\section{Conclusion}

We presented a new composite material that has efficient magnetic and plasmonic properties. It can efficiently absorb laser radiation and generate heat. Targeted delivery of particles with the help of an external magnet was demonstrated followed by the selected photothermal destruction of individual cells. It is important milestone for development of better materials for photothermal cancer treatment in humans, since magnetic control might be employed to concentrate photothermal material in the tumor and thus increase selectivity of heating the tumor vs. heating surrounding healthy tissue.

\section{Experimental section}

\section{Gold nanocages}

The $42 \mathrm{~nm}$ AuNCs were prepared using a galvanic replacement reaction between $36 \mathrm{~nm} \mathrm{Ag}$ nanocubes and $\mathrm{HAuCl}_{4}$ in an aqueous environment by following previously published protocol. ${ }^{10}$ The nanocages were purified by centrifugation at $10000 \mathrm{rpm}$ for $10 \mathrm{~min}$, washed twice with water, and, finally, redispersed in water at $1.8 \mathrm{nM}$ as the stock solution. The UV-vis spectra were measured using a Perkin-Elmer Lambda 750 spectrophotometer (Waltham, MA), and the LSPR peak of Au nanocages was determined to be at $785 \mathrm{~nm}$.

\section{Creation of the composite material - magnetic beads coated with Au nanocages}

To coat the magnetic beads with Au nanocages, $100 \mu \mathrm{l}$ of $10 \%$ (v/ v) of $\mathrm{Au}$ nanocages suspension was mixed with magnetic microbeads extracted from $300 \mu \mathrm{l}$ of $2.5 \%(\mathrm{w} / \mathrm{v})$ suspension of AMS-40-10H SPHERO ${ }^{\mathrm{TM}}$ amino smooth surface magnetic particles with high iron content. The suspensions were thoroughly mixed and the particles were allowed to electrostatically interact with each other ${ }^{20}$ for one hour at room temperature. The magnetic beads were captured inside of the glass vial using an external magnet, and all the nanocages that were not attached to the microbeads were washed away.

\section{Thermosensitive solution}

Elastin like polypeptides are derived from natural elastin and are composed of repeated blocks of penta-peptide, Val-Pro-Gly$\mathrm{X}$-Gly, where $\mathrm{X}$ is a guest residue that can be any amino acid except proline. At lower critical solution temperature (LCST), ELPs undergoes a phase transition. When the temperature is lower than LCST, ELPs stay soluble in aqueous solution, and with the temperature above LCST, ELPs self-assemble and become insoluble. Elastin like peptide sequence V50 (V: VPGVG) has been produced in the lab and used in this work. The generation of the ELP plasmid and expression and purification of the ELP were based on the methods by Koria et al. ${ }^{35}$

\section{Laser and optical fiber}

A fiber-coupled laser diode from ThorLabs with wavelength of $808 \mathrm{~nm}$ and nominal maximum power of $30 \mathrm{~mW}$ was used on its continuous wave (CW) mode. A single mode freshly cleaved optical fiber with a core diameter of $8 \mu \mathrm{m}$ was used in the experiments.

\section{Trypan blue staining of the fibroblast cells}

The trypan blue was purchased from Sigma-Aldrich, and was used directly without further purification. For staining, $100 \mu \mathrm{l}$ of trypan blue solution (0.4\%) was added into wells containing fibroblast cells to the final concentration of $0.04 \%$ and were allowed to mix for three minutes before starting the experiment. As a result, under visible light, live cells are transparent while dead cells become blue.

\section{Conflicts of interest}

There are no conflicts of interest to declare.

\section{Acknowledgements}

Gold nanocages were provided by Prof. Younan Xia and his student Bo Pang. This work has been partially conducted using facilities of the Florida Center of Excellence for Drug Discovery \& Innovation at the University of South Florida and USF 
Nanotechnology Research \& Education Center. SC was supported by the USF Signature Research Doctoral Fellowship.

\section{References}

1 J. Y. Chen, C. Glaus, R. Laforest, Q. Zhang, M. X. Yang, M. Gidding, M. J. Welch and Y. N. Xia, Small, 2010, 6, 811.

2 J. E. Kennedy, Nat. Rev. Cancer, 2005, 5, 321.

3 S. G. Bown, World J. Surg., 1983, 7, 700.

4 D. E. J. G. J. Dolmans, D. Fukumura and R. K. Jain, Nat. Rev. Cancer, 2003, 3, 380.

5 R. R. Anderson and J. A. Parrish, Science, 1983, 220, 524.

6 X. H. Huang, P. K. Jain, I. H. El-Sayed and M. A. El-Sayed, Laser Med Sci., 2008, 23, 217.

7 B. C. Wilson and M. S. Patterson, Phys. Med. Biol., 1986, 31, 327.

8 X. Huang and M. A. El-Sayed, Alexandria Journal of Medicine, 2011, 47, 1.

9 (a) A. O. Govorov and H. H. Richardson, Nano Today, 2007, 2, 30; (b) G. Baffou, R. Quidant and C. Girard, Appl. Phys. Lett., 2009, 94, 153109; (c) J. Chen, F. Saeki, B. J. Wiley, H. Cang, M. J. Cobb, Z. Y. Li, L. Au, H. Zhang, M. B. Kimmey, X. D. Li and Y. N. Xia, Nano Lett., 2005, 5, 473.

10 S. E. Skrabalak, L. Au, X. D. Li and Y. N. Xia, Nat. Protoc., 2007, 2, 2182.

11 L. Cheng, C. Wang, L. Z. Feng, K. Yang and Z. Liu, Chem. Rev., 2014, 114, 10869.

12 (a) W. Brullot, V. K. Valev and T. Verbiest, Nanomed. Nanotechnol., 2012, 8, 559; (b) C. S. Levin, C. Hofmann, T. A. Ali, A. T. Kelly, E. Morosan, P. Nordlander, K. H. Whitmire and N. J. Halas, ACS Nano, 2009, 3, 1379; (c) Z. Fan, D. Senapati, S. A. Khan, A. K. Singh, A. Hamme, B. Yust, D. Sardar and P. C. Ray, Chem. -Eur. J., 2013, 19, 2839.

13 W.-P. Li, P.-Y. Liao, Ch.-H. Su and Ch.-Sh. Yeh, J. Am. Chem. Soc., 2014, 36(28), 10062.

14 L. Cheng, K. Yang, Y. Li, J. Chen, Ch. Wang, M. Shao, Sh.-T. Lee and Zh. Li, Angew. Chem., Int. Ed., 2011, 50, 7385.

15 L. Cheng, K. Yang, Y. Li, X. Zeng, M. Shao, S. T. Lee and Z. Liu, Biomaterials, 2011, 33, 2215.
16 A. J. Rosengart, M. D. Kaminski, H. T. Chen, P. L. Caviness, A. D. Ebner and J. A. Ritter, J. Magn. Magn. Mater., 2005, 293, 633.

17 S. C. McBain, H. H. P. Yiu and J. Dobson, Int. J. Nanomed., 2008, 3, 169.

18 M. Shinkai, J. Biosci. Bioeng., 2002, 94, 606.

19 A. K. Gupta and M. Gupta, Biomaterials, 2005, 26, 3995.

20 M. Kanahara, M. Shimomura and H. Yabu, Soft Matter, 2014, 10, 275.

21 S. Cheemalapati, M. Ladanov, B. Pang, Y. Yuan, P. Koria, Y. Xia and A. Pyayt, Nanoscale, 2016, 8(45), 18912-18920.

22 H. C. Huang, P. Koria, S. M. Parker, L. Selby, Z. Megeed and K. Rege, Langmuir, 2008, 24, 14139.

23 H. M. Pollock and A. Hammiche, J. Heat Transfer, 2001, 34, R23.

24 N. Akino, T. Kunugi, K. Ichimiya, K. Mitsushiro and M. Ueda, J. Heat Transfer, 1989, 111, 2.

25 A. M. Chaudhari, T. M. Woudenberg, M. Albin and K. E. Goodson, J. Microelectromech. Syst., 1998, 7, 345.

26 K. L. Davis, K. L. K. Liu, M. Lanan and M. D. Morris, Anal. Chem., 1993, 65, 293.

27 M. E. Lacey, A. G. Webb and J. V. Sweedler, Anal. Chem., 2000, 72, 4991.

28 D. Ross, M. Gaitan and L. E. Locascio, Anal. Chem., 2001, 73, 4117.

29 H. Watzig, Chromatographia, 1992, 33, 445.

30 G. Baffou, C. Girard and R. Quidant, Phys. Rev. Lett., 2010, 104, 136805.

31 G. Baffou, M. Kreuzer, F. Kulzer and R. Quidant, Opt. Express, 2009, 17, 3291.

32 G. Baffou, P. Bon, J. Savatier, J. Polleux, M. Zhu, M. Merlin, H. Rigneault and S. Monneret, ACS Nano, 2012, 6, 2452.

33 M. T. Carlson, A. Khan and H. H. Richardson, Nano Lett., 2011, 11, 1061.

34 L. B. Lowe, S. H. Brewer, S. Kramer, R. R. Fuierer, G. G. Qian, C. O. Agbasi-Porter, S. Moses, S. Franzen and D. L. Feldheim, J. Am. Chem. Soc., 2003, 125, 14258.

35 P. Koria, H. Yagi, Y. Kitagawa, Z. Megeed, Y. Nahmias, R. Sheridan and M. L. Yarmush, Proc. Natl. Acad. Sci. U. S. A., 2011, 108, 1034. 